\title{
A comparative analysis between FEM and boundary methods in bending of plates
}

\section{S. HERNÁNDEZ}

Dept. de Estructuras, University of Zaragoza, Spain

\section{J. DÍAZ DEL VALLE and A. SAMARTÍN}

University of Santander, Spain

\section{INTRODUCTION}

Two different methods of analysis of plate bending are discussed in this paper. The plate behaviour is assumed to be represented by using the linear thin plate theory where the Poisson-Kirchoff assumption holds. Then the main structural results of the bending of the plate can be obtained by solving the following boundary value problem:

$$
\begin{gathered}
L(\omega)+b=0 \text { for }\left(x_{1}, x_{2}\right) A \\
\mathbf{B}(\omega) M(\omega)+\mathbf{t}=\mathbf{0} \text { for }\left(x_{1}, x_{2}\right) \bar{A}
\end{gathered}
$$

where $\left(x_{1}, x_{2}\right)$ represents a co-ordinate system in the plane middle surface of the plate, $A$ and $\bar{A}$ are the plate domain and its boundary (adherence) respectively.

$L$ is a fourth order differential operator. In the case of an orthotropic homogeneous plate this operator becomes:

$$
k_{i j} \frac{\partial^{4}}{\partial x_{i}^{2} \partial x_{j}^{2}}=k_{11} \frac{\partial^{4}}{\partial x_{1}^{4}}+2 k_{12} \frac{\partial^{4}}{\partial x_{1}^{2} \partial x_{2}^{2}}+k_{22} \frac{\partial^{4}}{\partial x_{2}^{4}}
$$

The use of the orthotropic plate as a modelling tool, particularly in relation to bridge decks is given among others in reference (1). $\omega=\left(x_{1}, x_{2}\right)$ is the deflection normal to the plate middle surface at point $\left(x_{1}, x_{2}\right)$, and $b=$ $b\left(x_{1}, x_{2}\right)$ is the corresponding distributed external forces.

At each point of the boundary $\bar{A}$ two conditions must be specific values (zero in the case of homogeneous boundary conditions).

The closed form solution of the problem given by equations (1) and (2) can be reached for only a very simple case. In general, a numerical solution must be considered, by the unknown function in the form:

$$
\omega \simeq \bar{\omega}=\sum_{1}^{n} a_{i} N_{i}
$$

where $N_{i}=N_{i}\left(x_{1}, x_{2}\right)$ are prescribed shape functions (subject to same broad conditions) and $a_{i}$ are unknown coefficients.

In the case of the boundary methods (Trefftz methods) the shape functions must satisfy the following conditions:

$$
L(\bar{\omega})=0
$$

or take into account the linearity of the operator $L$ :

$$
\text { - } L\left(N_{i}\right)=0
$$

Accepted January 1984. Discussion closes August 1984.
In general for arbitrary values of $a_{i}$ the function $\bar{\omega}$ does not fulfil the boundary conditions, i.e.

$$
\mathbf{B}(\bar{\omega})=\mathbf{0}
$$

The coefficients $a_{i}$ can be found by satisfying the condition (4) along the boundary in some sense (locally, average, etc.).

In reference (2) several methods to ubtain the coefficients $a_{i}$ are shown. Namely they can be divided into two groups. The first corresponds to the weighted residues, i.e. the $n$ equations to find the coefficients $a_{i}$ are:

$$
\oint_{\bar{A}} \mathbf{W} \cdot \mathbf{B}(\bar{\omega}) \mathrm{d} s=\mathbf{0}
$$

where $\mathrm{W}$ is a vector of dimension $(n \times 2)$ containing the weighted functions and $\mathrm{d} s$ is the differential length of $\bar{A}$. The second group of methods are given from the stationary condition of the functional:

$$
\Pi=\int_{\bar{A}} \mathbf{B}^{T} \mathbf{K} \mathbf{B} \mathrm{d} s
$$

where $\mathbf{K}=\operatorname{diag}\left[K_{1}(s), K_{2}(s)\right]$ is a diagonal weighted error matrix.

The stationarity condition of (6) corresponds to the values of $a_{i}$ satisfying the simultaneous linear equations:

$$
\frac{\partial \Pi}{\partial a_{i}}=0 \quad(i=1,2, \ldots, n)
$$

Both kinds of numerical methods carry out a convergence process in mean sense; because of that it is not possible to find the singularities noticed by Williams. ${ }^{3}$

The solution given by Samartin ${ }^{4}$ corresponding to a rectangular orthotropic plate has been extended in ref. 2 in order to consider skew orthotropic plates, i.e. simply supported plates along two parallel opposite edges and the two remaining edges with arbitrary geometry and boundary conditions. Different weighted functions $\mathbf{W}$ and error matrices $\mathbf{K}$ have been considered and compared, ${ }^{2}$ for the special simple case of a parallelogram plate. From this comparison, the solution obtained by the functional use of the matrix $\mathbf{K}(s)$ and the corresponding dual values to the essential and natural specified boundary conditions has been proved to give the best results. In this sense this paper 
represents an extension of ref. 5, since we are going to consider non-symmetrical shapes, other loading cases and boundary conditions. Moreover, the rule to identify the best weighting functions is also given. A comparison between this boundary method and the FEM is carried out. Both types, conforming and non-conforming finite elements, are used in this comparative study.

\section{GEOMETRY AND LOADING CASES}

Two plate geometries have been considered, namely, parallelogram plates with skew angles $(\alpha) 30^{\circ}$ and $45^{\circ}$ and different ratios between the lengths $L_{2}$ and $L_{1}$ (Fig. 1). The boundary conditions have been simply supported along the sides of length $L_{2}$ and two cases of boundary conditions along the two other edges are simply supported and free. The loading cases analysed are summarised as follows (see Fig. 2):

(a) Uniform load of intensity, $1 \mathrm{tm}^{-2}$.

(b) Concentrated point load at middle point of the plate of intensity, $100 \mathrm{t}$.

(c) Tranversal knife load acting parallel to the supports (sides of length $L_{2}$ ) along the central cross-section. Intensity, $10 \mathrm{tm}^{-1}$.

(d) Longitudinal knife load normal to the supports and passing by the middle point of the plate. Intensity, $10 \mathrm{tm}^{-1}$.

(e) Longitudinal knife load parallel to the free edges (sides of length $L_{1}$ ) and passing by the middle point of the plate. Intensity, $10 \mathrm{tm}^{-1}$.

All the analysed plates have been assumed to be isotropic with Young's modulus: $E=2.1 \times 10^{6} \mathrm{tm}^{-2}$ and thickness $t=1.00 \mathrm{~m}$.

The Poisson ratio has been assumed to be $\nu=0.15$ for the plate with two free edges and $\nu=0.20$ for the plates with all edges simply supported. For these plates constant $D$ is defined as follows:

$$
D=\frac{E t^{3}}{12\left(1-\nu^{2}\right)}
$$

\section{SKEW PLATES WITH TWO FREE EDGES}

Plates with skew angle $\alpha=30^{\circ}$

In the Boundary Method (BM) the number of shape functions used has been between 16 and 24, depending on
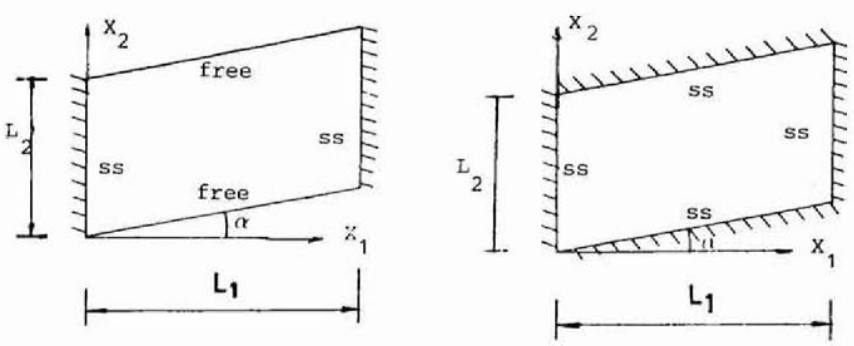

\begin{tabular}{|c|c|c|c|}
\hline Angle $\alpha$ & \multicolumn{3}{|c|}{$L_{2}$} \\
\hline $30^{\circ}$ & 1.5 & 1 & 0.5 \\
\hline $45^{\circ}$ & - & 1 & - \\
\hline
\end{tabular}

\begin{tabular}{|c|c|}
\hline Angle $\alpha$ & $\frac{L_{2}}{L_{1}}$ \\
\hline $30^{\circ}$ & 0,66 \\
\hline $45^{\circ}$ & 1 \\
\hline
\end{tabular}

Figure 1. Geometry and boundary conditions of the plate

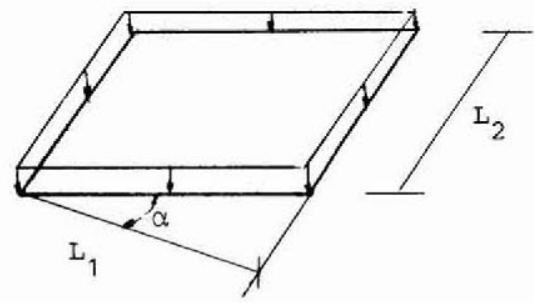

Case a)
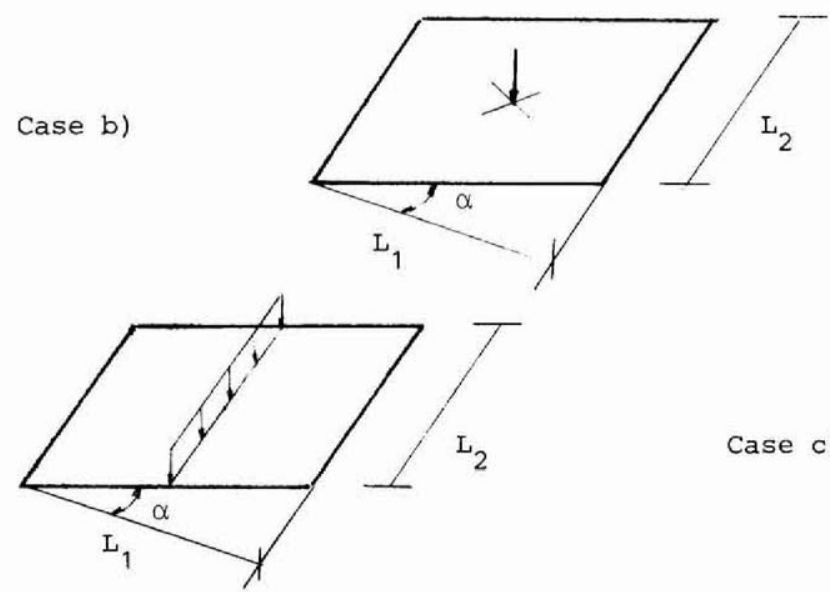

Case c)
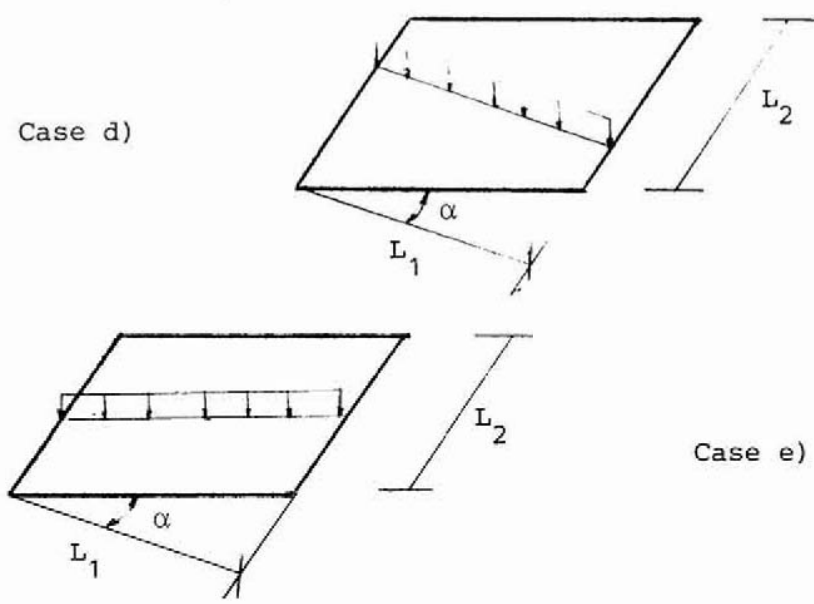

Case e)

Figure 2. Loading case

the convergence requirements. In Table 1 the different ratios $L_{2} / L_{1}$ considered are given.

The Finite Element Method is applied by using the program SAPIV, with a finite element mesh of 64 elements, as represented in Fig. 3.

The results are vertical displacement, $w(\mathrm{~cm})$, and the longitudinal bending moment, $m_{11}(\mathrm{mt} / \mathrm{m})$, at the plate middle point.

The comparison between these two results are given in Table 1.

Plates with skew angles $\alpha=45^{\circ}$

The same results defined in the previous section are used to observe the computational efficiency of each method. In order to compare a similar number of simultaneous linear equations to be solved in each method, different finite element meshes (Fig. 4) have been considered. The results obtained are summarised in Table 2 for the particular geometric case of the plate $L_{2}: L_{1}=1$.

It is interesting to point out the greater differences between the deflection values and the bending moments. The explanation for this situation may be such that the 
Table 1. Central deflection $\omega(\mathrm{cm})$ and longitudinal bending moment $\left(m_{11}(\mathrm{mt} / \mathrm{m})-\right.$ skew plate $\left.\alpha=30^{\circ}\right)$

\begin{tabular}{|c|c|c|c|c|c|c|c|c|c|c|c|c|}
\hline \multirow{4}{*}{$\begin{array}{l}\text { Method } \\
\text { d.o.f. } \\
\text { Loading case }\end{array}$} & \multicolumn{4}{|c|}{$L_{2} / L_{1}=0.5$} & \multicolumn{4}{|c|}{$L_{2} / L_{1}=1$} & \multicolumn{4}{|c|}{$L_{2} / L_{1}=1.5$} \\
\hline & \multicolumn{2}{|c|}{$\mathrm{BM}$} & \multicolumn{2}{|c|}{ FEM } & \multicolumn{2}{|c|}{ BM } & \multicolumn{2}{|c|}{ FEM } & \multicolumn{2}{|c|}{$\mathrm{BM}$} & \multicolumn{2}{|c|}{ FEM } \\
\hline & \multicolumn{2}{|c|}{24} & \multicolumn{2}{|c|}{225} & \multicolumn{2}{|c|}{16} & \multicolumn{2}{|c|}{225} & \multicolumn{2}{|c|}{16} & \multicolumn{2}{|c|}{225} \\
\hline & $\omega$ & $m_{11}$ & $\omega$ & $m_{11}$ & $\omega$ & $m_{11}$ & $\omega$ & $m_{11}$ & $\omega$ & $m_{11}$ & $\omega$ & $m_{11}$ \\
\hline (a) & 0.85 & 41.2 & 0.92 & 47.4 & 0.81 & 40.7 & 0.84 & 40.3 & 0.83 & 41.2 & 0.80 & 39.6 \\
\hline (b) & 0.80 & 49.0 & 0.94 & 47.5 & 0.44 & 33.6 & 0.46 & 27.5 & 0.37 & 29.9 & 0.36 & 21.6 \\
\hline (c) & 1.05 & 51.3 & 1.17 & 47.2 & 0.55 & 30.0 & 0.57 & 29.1 & 0.45 & 24.5 & 0.43 & 22.3 \\
\hline (d) & 0.80 & 43.6 & 0.84 & 52.8 & 0.76 & 43.4 & 0.79 & 45.0 & 0.77 & 43.2 & 0.73 & 40.0 \\
\hline
\end{tabular}

Table 2. Central deflection $\omega(\mathrm{cm})$ and longitudinal bending moment $\left(m_{11}(\mathrm{mt} / \mathrm{m})-\right.$ skew plate $\left.\alpha=45^{\circ}, L_{2} / L_{1}=1\right)$

\begin{tabular}{|c|c|c|c|c|c|c|c|c|}
\hline \multirow{3}{*}{$\begin{array}{l}\text { Method } \\
\text { d.o.f. } \\
\text { Loading case }\end{array}$} & \multicolumn{2}{|c|}{ BM } & \multicolumn{2}{|c|}{ FEM $(2 \times 2)$} & \multicolumn{2}{|c|}{ FEM $(4 \times 4)$} & \multicolumn{2}{|c|}{ FEM $(8 \times 8)$} \\
\hline & \multicolumn{2}{|c|}{20} & \multicolumn{2}{|c|}{21} & \multicolumn{2}{|c|}{65} & \multicolumn{2}{|c|}{225} \\
\hline & $\omega$ & $m_{11}$ & $\omega$ & $m_{11}$ & $\omega$ & $m_{11}$ & $\omega$ & $m_{11}$ \\
\hline (a) & 0.71 & 34.6 & 0.63 & 19.5 & 0.92 & 37.5 & 1.01 & 31.7 \\
\hline (b) & 0.27 & 22.0 & 0.39 & 9.5 & 0.51 & 17.0 & 0.55 & 23.9 \\
\hline (c) & 0.50 & 27.8 & 0.70 & 16.0 & 0.73 & 24.6 & 0.80 & 29.7 \\
\hline (d) & 0.39 & 20.3 & 0.36 & 8.6 & 0.55 & 17.3 & 0.41 & 16.7 \\
\hline (e) & 0.56 & 32.2 & 0.70 & 16.0 & 0.87 & 26.8 & 0.94 & 32.8 \\
\hline
\end{tabular}

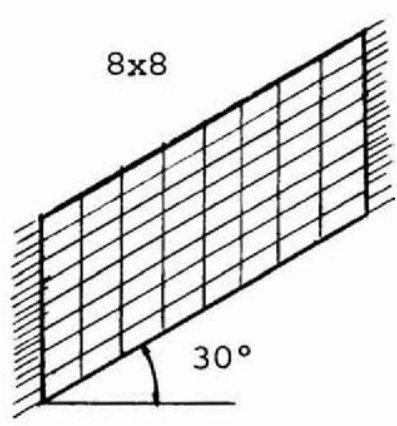

Figure 3. Finite element mesh
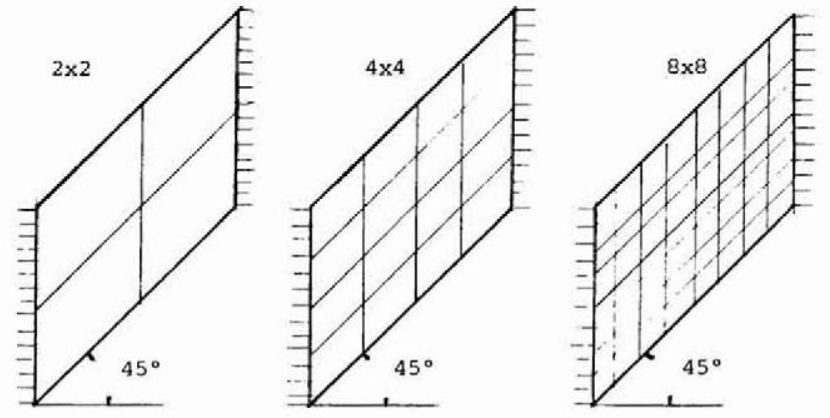

Figure 4. Different finite element meshes

error function to be minimised includes only bending moments and Kirchoff shears, i.e. the deflections are not considered in the functional of the boundary conditions.

\section{SKEW PLATES WITH SIMPLY SUPPORTED EDGES}

It is also interesting to compare the two methods FEM and BM in the analysis of plates with other boundary condi- tions along the sides $L_{1}$ different to the free edge. The case considered was that of a plate simply supported along its contour under uniform load of intensity $q$. The BM solution is compared with that obtained by the use of the conforming triangular hyperelement described in ref. 7 . This hyperelement assumed a $7^{\circ}$ polynomial expansion for the deflection, i.e. each triangle represents $36^{\circ}$ of freedom (d.o.f.) according to Fig. 5. Also the results obtained by Jensen ${ }^{8}$ using a very fine finite difference mesh are included for comparison purposes. The two cases studied are:

(a) Plate with a skew angle $\alpha=30^{\circ}$ and $L_{2}: L_{1}=0.66$. The results obtained for the displacement $\omega$ and maximum bending moment $m$ at the plate middle point are given in Table 3.

These results show a good agreement between the BM (with six Fourier terms, i.e. $n=24$ ) and the 'exact' values

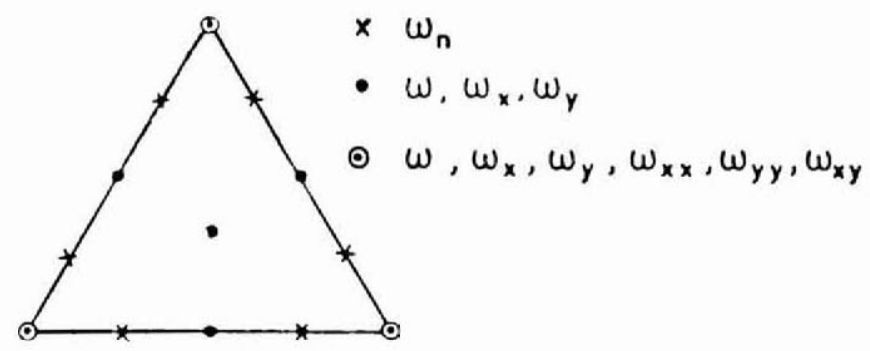

Figure 5. Hyperelement 7 th degree polynomial

Table 3. Central deflection $\omega(\mathrm{m})$ and maximum bending $(\mathrm{mt} / \mathrm{m})$

\begin{tabular}{lclll}
\hline & BM & \multicolumn{1}{c}{ Jensen } & \multicolumn{1}{c}{ FEM } & \\
\hline $\begin{array}{l}\text { Central deflection } \\
\text { Maximum bending }\end{array}$ & 0.0096 & 0.01046 & 0.01040 & $\times q L_{1}^{4} / D$ \\
moment & 0.0972 & 0.0968 & 0.0970 & $\times q L_{1}^{2}$ \\
\hline
\end{tabular}


Table 4. Central deflection $\omega(\mathrm{m})$ and maximum bending $(\mathrm{mt} / \mathrm{m})$

\begin{tabular}{lllll}
\hline & \multicolumn{1}{c}{ BM } & Jensen & FEM & \\
\hline Central deflection & 0.00922 & 0.00938 & 0.00930 & $\times q L_{1}^{4} / D$ \\
Maximum bending & 0.0853 & 0.00898 & 0.00881 & $\times q L_{1}^{2}$ \\
\hline
\end{tabular}

obtained by the Jensen analysis. The agreement reaches $92 \%$ for the deflection and $100.5 \%$ for the bending moment. In the FEM the number of d.o.f. is 55 and in the Jensen analysis this number is even greater.

(b) Plate with a skew angle $\alpha=45^{\circ}$ and $L_{2}: L_{1}=1$. In this situation Table 4 shows a very good agreement among the three analyses. It may be worthwhile to notice the differences of $95 \%$ for the bending moments and $98.3 \%$ for the deflections between the values obtained from BM and the 'exact' ones given for the two other methods.

\section{CONCLUSIONS}

From the previous analysis the following provisional conclusions can be drawn:

(1) The BM based in a weighted mean square error technique produced good results for the problem of plate bending.

(2) The functions used in the BM can be the trigonometric exponential ones corresponding to the Levy solution of the rectangular plate.

(3) The computational effort demanded in the BM is smaller than the one needed in a FEM analysis for the same level of accuracy.

(4) The general application of the FEM cannot be matched by the BM. Particularly, different types of geometry (plates of arbitrary geometry) need a similar but not identical treatment in the BM. However, this loss of generality is counterbalanced by the computational efficiency gained in the BM in the solution achievement.

(5) The proposed BM analysis may be ameliorated by the use of similar techniques as the ones developed in the Boundary Element Methods. Further studies in this direction are now in progress.

\section{REFERENCES}

1 Samartín, A. Cálculo de estructuras de puentes de hormigón, Ed. Rueda, Madris 1983

2 Hernández, S. Aplicación de los métodos de contorno a la flexión de placas de forma arbitraria, Universidad de Santander, 1982

3 Williams, M. L. Surface stress singularities resulting from various boundary conditions in angular corners of plates under bending, U.S. Nat. Cong. Appl. Mech. Illinois, Inst. of Technology, 1951, 325

4 Samartín, A. Reparto transversal de la sobrecarga en tableros de puentes, Hormigón y Acero, 1977

5 Morley, L. S. D. Bending of a simply supported rhombic plate under uniform normal loading, Q. J. Mech. Applied Maths. 1962, XV (4)

6 Clough, R. W. and relippa, C. A. A refined quadrilateral element for analysis of plate bending, Proc. 2nd Conf. on Matrix Methods in Structural Mechanics, Wright Patterson AFB, Ohio, 1968

7 Díaz del Valle, J. and Samartín, A. Hiperelementos finitos aplicados a la flexión de placas delgadas, Universidad de Santander, 1980

8 Jensen, V. P. Univ. Illinois Bull. 1947, 332 and 369

\section{BIBLIOGRAPHY}

Bathe, K. J. and Wilson, E. SAP IV (a structural analysis program for static and dynamic response of linear systems), University of California, 1974 